\title{
Wide and scalable field-of-view Talbot-grid-based fluorescence microscopy
}

\author{
Shuo Pang, ${ }^{1, *,+}$ Chao Han, ${ }^{1,+}$ Mihoko Kato, ${ }^{2}$ Paul W. Sternberg, ${ }^{2}$ and Changhuei Yang ${ }^{1}$ \\ ${ }^{1}$ Department of Electrical Engineering, California Institute of Technology, Pasadena, California 91125, USA \\ ${ }^{2}$ Howard Hughes Medical Institute and Division of Biology, California Institute of Technology, Pasadena, California 91125, USA \\ *Corresponding author: spang@caltech.edu
}

Received September 18, 2012; revised October 24, 2012; accepted November 5, 2012;

posted November 7, 2012 (Doc. ID 176512); published November 30, 2012

\begin{abstract}
Here we report a low-cost and simple wide field-of-view (FOV) on-chip fluorescence-imaging platform, termed fluorescence Talbot microscopy (FTM), which utilizes the Talbot self-imaging effect to enable efficient fluorescence imaging over a large and directly scalable FOV. The FTM prototype has a resolution of $1.2 \mu \mathrm{m}$ and an FOV of $3.9 \mathrm{~mm} \times 3.5 \mathrm{~mm}$. We demonstrate the imaging capability of FTM on fluorescently labeled breast cancer cells (SK-BR-3) and human embryonic kidney 293 (HEK) cells expressing green fluorescent protein. @ 2012 Optical Society of America
\end{abstract}

OCIS codes: $180.2520,110.6760$

Because of the high specificity and high sensitivity of fluorescent probes, fluorescence microscopy plays a vital role in modern clinical diagnosis and biological research. However, because of its limited field-of-view (FOV), size, and cost, conventional microscopy is becoming a bottleneck in rapidly emerging and evolving areas such as large-scale genome screening [1], point-of-care diagnosis, and long-term cell imaging [2]. In recognition of these disadvantages, several technologies have recently been developed to enable high throughput screening for diagnosis and gene activity quantification [2,3]. These systems work by optimizing the transport of samples under the conventional microscope's FOV to increase the throughput. In essence, these systems achieve an effectively larger FOV by lengthening the imaging time- - the scaling is linearly proportionate.

More fundamentally, the direct FOV of the conventional microscope objective is inversely related to the resolution. As a reference point, a standard $20 \times$ microscope objective (Plan, Olympus) has a resolution of $\sim 0.8 \mu \mathrm{m}$ and an FOV of $\sim 5 \times 10^{-2} \mathrm{~mm}^{2}$. The link between the FOV and the resolution is an inherent disadvantage of the conventional microscope and is primarily due to the aberrations associated with optical elements in the microscope's objective. Previous novel attempts at breaking this link have been reported [4,5]. However, none has reported resolution that is $\sim 1 \mu \mathrm{m}$ or better.

Here we present a wide FOV fluorescence microscopy method, termed fluorescence Talbot microscopy (FTM). Unlike for conventional fluorescence microscopy, the FOV for FTM can be arbitrarily scaled up without incurring a proportionate imaging time increase or sacrifice of resolution. Our FTM prototype has a demonstrated resolution of $1.2 \mu \mathrm{m}$ and an FOV of $3.9 \mathrm{~mm} \times 3.5 \mathrm{~mm}$.

The FTM prototype utilizes a microlens grid (SUSS, 1800407) to transform the incident coherent plane wave with wavelength $\lambda$ into a grid of tightly focused light spots at the lenses' focal distance $Z_{f}=90 \mu \mathrm{m}$. The separation between adjacent focal spots is equal to the microlens grid pitch $d=30 \mu \mathrm{m}$. This light grid then propagates and reforms into focused light spots at distance of $z=n Z_{T}$, where $n$ is an integer and $Z_{T}=2 d^{2} / \lambda$ is defined as the Talbot length. This self-imaging phenomenon is known as the Talbot effect []ㅡ. For the FTM prototype, we employ a laser (Fibertech, $\lambda=488 \mathrm{~nm}, 30 \mathrm{~mW}$ ) as the light source. This allows us to create a focused light spot grid (at the first Talbot image, $n=1$ ) at $Z_{T}=3.7 \mathrm{~mm}$ below the microlens grid. The Talbot effect homogenizes the intensity of the periodic input field. In a prior work, we have demonstrated that this Talbot focal grid has good focusing characteristics ( $\sim 1 \mu \mathrm{m}$ spot diameter) and spot power uniformity [7].

For this current system, we project the grid of Talbot focal spots onto the target sample. The sample rests on a filter-coated metal-oxide-semiconductor (CMOS) image sensor chip [Fig. 1(a)]. The fluorescence emissions associated with each focal spot are collected by the sensor chip and are composed into a sparsely illuminated, and therefore sparsely sampled, fluorescence image. By angularly tilting the incident laser [Fig. 1(b)], we can then raster scan the Talbot focal spot grid across the sample and collect a sequence of sparse images. Finally, we reconstruct a filled-in high-resolution fluorescence image [Fig. $\underline{1(\mathrm{c})}$ ].

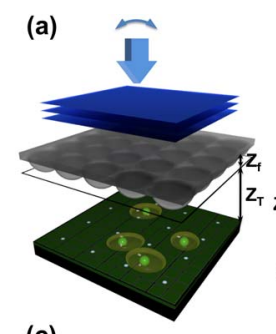

(c) (b)

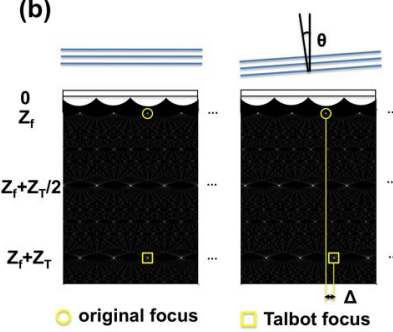

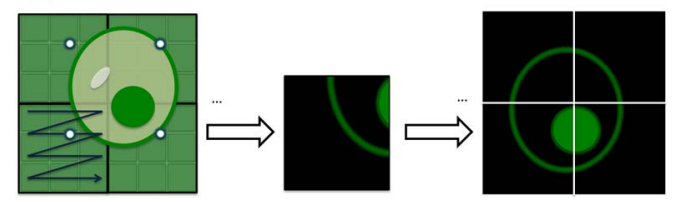

Fig. 1. (Color online) FTM operating principle. (a) The schematic diagram of the FTM system: the microlens grid creates the original focal grid at $Z_{f}$. The sample on a filter-coated CMOS sensor is located at the Talbot length $Z_{T}$ away from the original focal grid. (b) As the plane wave is tilted by a small angle $\theta$, the Talbot focal grid laterally shifts by $\Delta$. (c) Scan and reconstruction method for the full-field image. 
The FTM system is distinct from previously described systems [구요. In those systems, a translational stage scans the sample, and an optical relay system collects the light. As a result, the system is inherently more costly and complex. The FTM method simplifies the system by taking advantage of a subtle property of the Talbot effect: the regenerated Talbot grid can laterally translate a significant amount in response to a small angular tilt of the input light field [Fig. 1(b)] [9]. Mathematically, the translation of the first Talbot image $(n=1), \Delta$, versus angular tilt, $\theta$, is given by

$$
\Delta=2 d^{2} \tan (\theta) / \lambda
$$

In the context of our prototype, an angular tilt of $8.2 \mathrm{mrad}$ will result in a $30 \mu \mathrm{m}$ lateral translation of the first Talbot grid to allow the full raster scan over the sample. The requisite raster scan for full sample coverage using the original focal grid is $0.33 \mathrm{rad}\left(31^{\circ}\right)$, which would significantly deteriorate image resolution. The pitch of the microlens grid ensures that the fluorescent signals from adjacent Talbot spots are well separable. This directon-chip collection eliminates the need for relay optics and circumvents the FOV limitation by the relay optics.

The FTM system includes a Talbot illuminator and the CMOS sensor [Fig 2(a)]. The CMOS sensor (Aptina, MT9P031I12STM) was coated with a green or a red filter. The optical densities of the filters at the excitation wavelength are $\sim 6$ and the filter thicknesses are $\sim 10 \mu \mathrm{m}[10]$. We established that the modified sensor sensitivity is $\sim 2 \times 10^{6}$ fluorophores $/ \mu \mathrm{m}^{2}$ for fluorescein illuminated with $488 \mathrm{~nm}$ laser at an intensity $\sim 10^{2} \mathrm{~W} / \mathrm{cm}^{2}$ with an integration time of $50 \mathrm{~ms}$. The Talbot illuminator creates the scanning Talbot grid for the sample. A laser beam from the single-mode fiber is collimated and then reflected by the microelectromechanical system (MEMS) mirror (Mirrorcle). A polarizing beam splitter and a quarter-wave plate minimize the intensity loss by the MEMS mirror. The beam is expanded to provide an angularly scannable illumination to the microlens grid [Fig. 2(b)].

The resolution, FOV, and collection efficiency of a conventional microscope objective are tied to each other. For FTM, the resolution depends on the Talbot focal spot size; the FOV is related to the extent of the focal spot grid and the sensor's dimension; the collection efficiency is related to the physical proximity of the fluorescence sites (a)

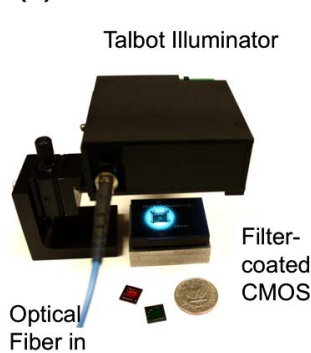

(b)

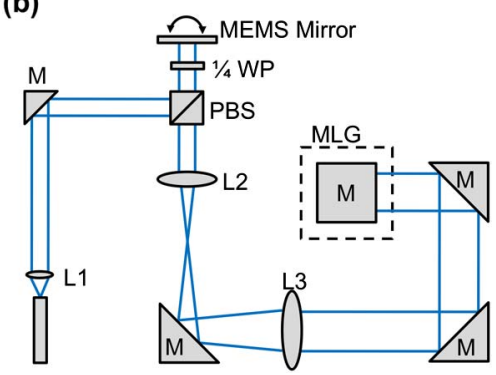

Fig. 2. (Color online) (a) FTM prototype: the filter coated CMOS sensor is placed beneath the Talbot illuminator. (b) Schematics of the Talbot illuminator (top view). L1, fiber collimator $f=13 \mathrm{~mm}$; L2,$f=10 \mathrm{~mm}$; L3, $f=50 \mathrm{~mm} ; M, 45^{\circ}$ mirrors; MLG, microlens grid; PBS, polarizing beam splitter; $1 / 4 \mathrm{WP}$, quarter-wave plate. to the sensor pixels. The FOV of the FTM design can be scaled up without limitations by simply using bigger sensor chips and larger lenslet grid.

The scalar wave simulations [11] showed that the original focal spot has a FWHM of $1.25 \mu \mathrm{m}$, and the FWHM at Talbot distance is $1.28 \mu \mathrm{m}$. It also verified that the fullrange scan angular tilt does not deteriorate the quality of Talbot focal spot [the spot never expand beyond a FWHM of $1.30 \mu \mathrm{m}$, as shown in Fig. 3(a)]. To evaluate the system resolution, we used FTM to image a resolution target mask on an aluminum layer deposited on the CMOS sensor patterned by focused ion beam (FIB) (FEI, Nova $600)$. The experiment established the resolution at $1.2 \mu \mathrm{m}$ [Figs. 3(b), 3(c)]. The ripple image artifacts, which are apparent in Fig. 2(b2) (especially visible for the $3.0 \mu \mathrm{m}$ lines), are attributable to the near-periodic detection signal variations when the transmitted Talbot spots fall between sensor pixels (pixel fill factor is not unity). This type of artifact is absent in fluorescence imaging as the

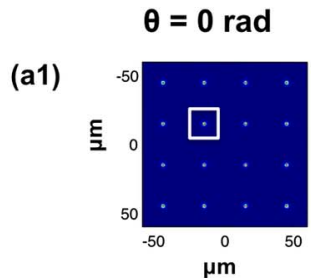

(a3)

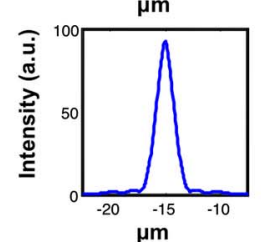

(a2)

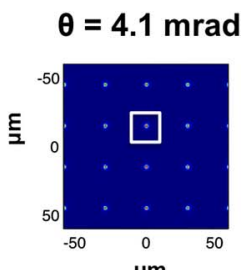

(a4)

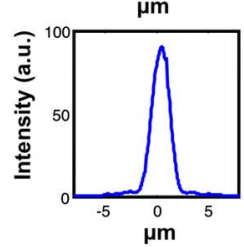

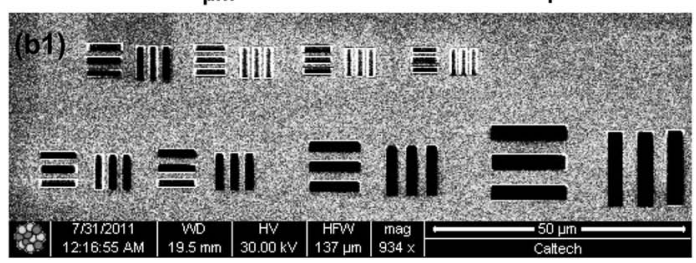
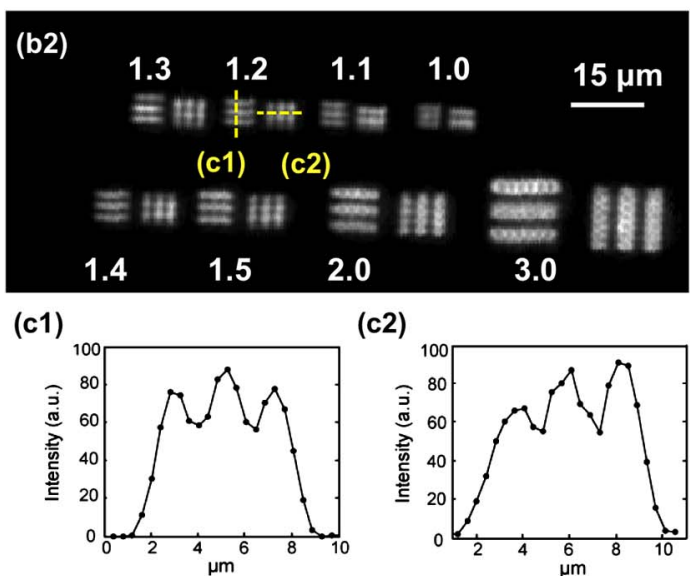

(c2)

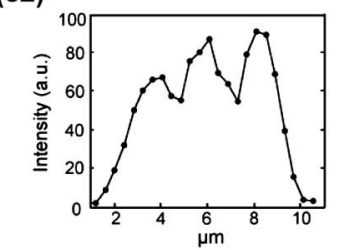

Fig. 3. (Color online) (a) Scalar wave simulations. (a1), (a2) First Talbot focal grid with incident angle of $0 \mathrm{rad}$ and $4.1 \mathrm{mrad}$, respectively. (a3), (a4) Intensity profile of the spot specified by the square in (a1) and (a2), respectively. (b) Resolution target with the linewidth from 3.0 to $1.0 \mu \mathrm{m}$ : (b1) an FIB image of the resolution target, (b2) the FTM image. (c) Cross-section line trace of the (c1) horizontal and (c2) vertical marks of $1.2 \mu \mathrm{m}$. 

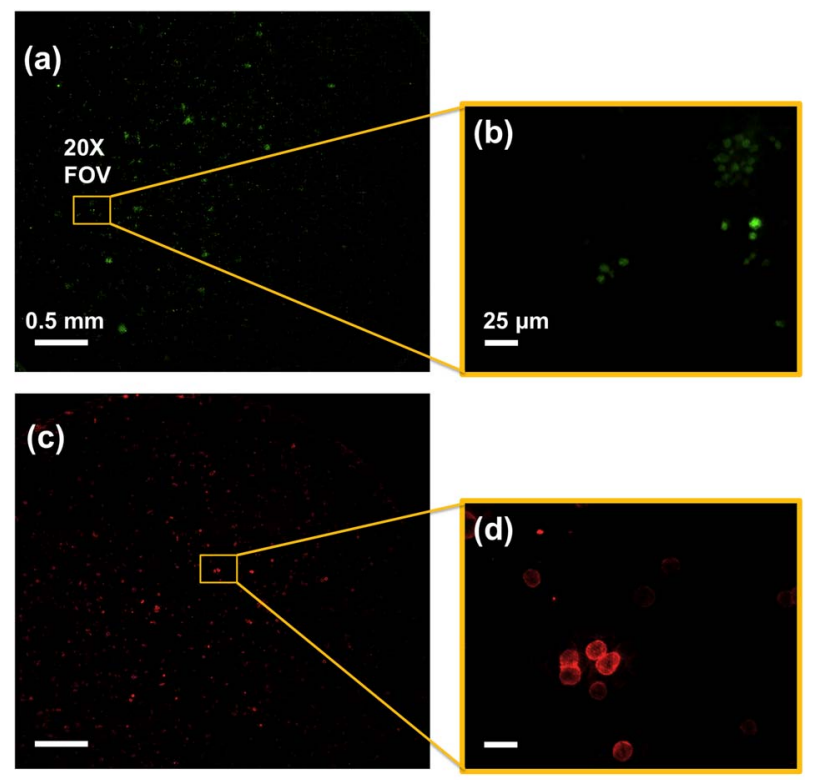

Uniform Illumination
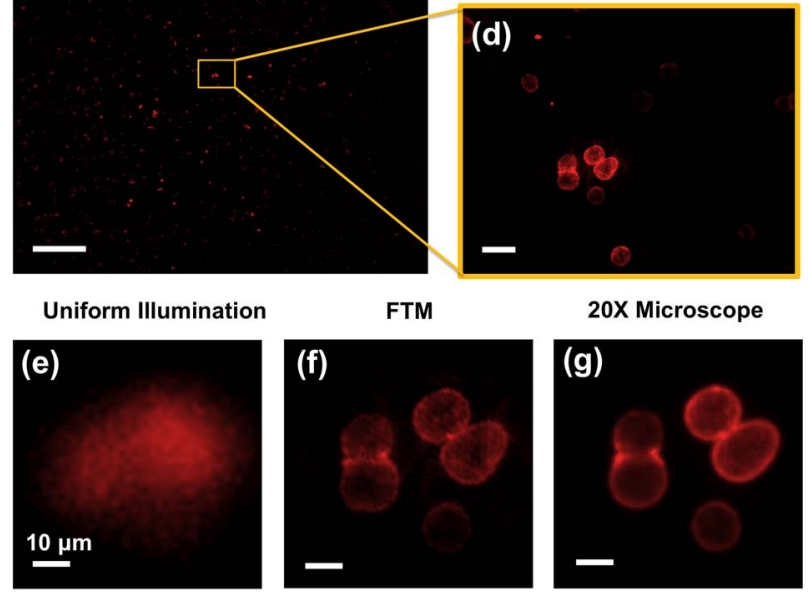

20X Microscope

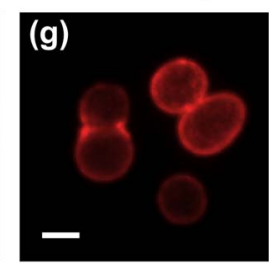

Fig. 4. (Color online) Full-field FTM images. (a) HEK cells expressing GFP in nuclei. (b) Magnified view of (a) corresponding to the FOV of a $20 \times$ microscope objective. (c), (d) SK-BR-3 cells with Her2 marker in the membrane labeled by Qdot625. (e), (f) Images of the same sample by on-chip detection using (e) uniform illumination (f) FTM, and (g) a conventional microscope with a $20 \times$ objective.

emitted fluorescence from a sample spread through the filter layer to $\sim 10 \times 10$ pixels for detection and thereby averaging out the fill factor associated artifacts.

We demonstrated the full-field FTM imaging of HEK cells expressing green fluorescence protein (GFP) in the nucleus [Figs. 4(a), 4(b)] and fixed fluorescence human cancer cells (SK-BR-3) with a cancer marker (Her2) in their membranes labeled by quantum dots (QDots 625) [Figs. 4(c), 4(d)]. Since the minimal discernible linewidth is $1.2 \mu \mathrm{m}$, we chose the scan step size to be $0.6 \mu \mathrm{m}$. Choosing a smaller step size will allow the system to closer approach the optimal resolution at the cost of acquisition time increase. The dwell time for each step depended on the fluorescent intensity from the sample. With approximately $1 \mu \mathrm{W}$ on each focal spot, the pixel dwell times for the HEK cell sample and the SK-BR-3 sample were set at 16 and $12 \mathrm{~ms}$, respectively, for total scan times of 50 and $38 \mathrm{~s}$, respectively. The data readout and storage time for the process, which was mainly limited by the sensor readout speed, was about 4-5 min. The full area of imaging was $3.9 \mathrm{~mm} \times 3.5 \mathrm{~mm}$ on the CMOS sensor. The reconstructed subimage from each focal spot consisted of $50 \times 50$ pixels. The full-field image combined the subimages of $128 \times 114$ Talbot focal spots from a stack of 2500 raw images. To show the benefit provided by FTM, we applied a uniform illumination without intervening microlenses and directly collected fluorescent signals; any structure that is finer than the fluorescent spread was indistinguishable [Fig. 4(e)]. Using the FTM method, the images were comparable with microscope images taken with a $20 \times$ objective [Figs. $4(\mathrm{f}), 4(\mathrm{~g})]$. The diagonal strip artifacts in Fig. 4(f) are likely attributable to long-time-scale gain fluctuation of the sensor circuit.

In summary, we have demonstrated a low-cost and simple wide FOV fluorescence imaging platform, FTM. The ability to easily expand its FOV is a significant advantage. In particular, the image acquisition time of conventional scanning systems scales linearly with the imaging area, while the image acquisition time for FTM would remain the same regardless of the area covered. When the FOV exceeds $\sim 20 \mathrm{~mm}^{2}$, the FTM method can be expected to start yielding faster imaging speed than a commercial high-throughput slide scanner, such as Hamamatsu nanoZoomer 2.0-HT. A narrow-bandwidth light source with high spatial coherence could replace the laser to further reduce the system cost.

This current FTM scheme is suited for fluorescence ePetri imaging [12]-self-imaging by smart petri dish housed in incubators. Commercialization efforts in other applications need to be cognizant of the direct contact between sample and sensor in this FTM implementation. It is possible to develop FTM schemes without this restriction.

We thank Danielle V. Bower (Biological Imaging Center, Caltech) for the help in sample preparation. This project is funded by NIH under the grant 1R01AI096226-01.

$\dagger$ These authors contributed equally to this work.

\section{References}

1. S. R. Collins, J. S. Weissman, and N. J. Krogan, Nat. Methods 6, 721 (2009).

2. B. Neumann, M. Held, U. Liebel, H. Erfle, P. Rogers, R. Pepperkok, and J. Ellenberg, Nat. Methods 3, 385 (2006).

3. K. H. Chung, M. M. Crane, and H. Lu, Nat. Methods 5, 637 (2008).

4. S. A. Arpali, C. Arpali, A. Coskun, H. H. Chiang, and A. Ozcan, Lab Chip 12, 4968 (2012).

5. A. F. Coskun, I. Sencan, T. W. Su, and A. Ozcan, Analyst 136, 3512 (2011).

6. W. D. Montgomery, J. Opt. Soc. Am. 57, 772 (1967).

7. J. G. Wu, G. A. Zheng, Z. Li, and C. H. Yang, Opt. Lett. 36, 2179 (2011).

8. A. Orth and K. Crozier, Opt. Express 20, 13522 (2012).

9. A. W. Lohmann and D. E. Silva, Opt. Commun. 2, 413 (1971).

10. S. Pang, C. Han, L. M. Lee, and C. H. Yang, Lab Chip 11, 3698 (2011).

11. J. W. Goodman, Introduction to Fourier Optics (Roberts, 1996).

12. G. A. Zheng, S. A. Lee, Y. Antebi, M. B. Elowitz, and C. H. Yang, Proc. Natl. Acad. Sci. USA 108, 16889 (2011). 